\title{
Colorectal neoplasm detection using virtual colonoscopy: a feasibility study
}

\author{
H M Fenlon, D P Nunes, P D Clarke, J T Ferrucci
}

\begin{abstract}
Background-Virtual colonoscopy is a potentially powerful tool for non-invasive colorectal evaluation. In vitro studies have established its accuracy in simulated polyp detection but little data exist regarding its use in clinical practice.

Aims-To evaluate the ability of virtual colonoscopy to detect colorectal cancers and polyps in patients with endoscopically proven colorectal neoplasms and to correlate the findings of virtual colonoscopy with those of conventional colonoscopy, surgery, and histopathology.

Patients-Thirty eight patients with endoscopic findings suggestive of colorectal carcinoma.

Methods-Virtual colonoscopy was performed using thin section helical computed tomography (CT) of the abdomen and pelvis after rectal insufflation of room air. Commercially available software was used to generate endoscopic "fly through" examinations of the colon from the CT data. Results were correlated with the findings of conventional colonoscopy and with the surgical and histopathological outcome in each case.

Results-Thirty eight pathologically proven colorectal cancers and 23 adenomatous polyps were present. On virtual colonoscopy, all cancers and all polyps measuring greater than $6 \mathrm{~mm}$ in size were identified; there were two false positive reports of polyps. On conventional colonoscopy, there was one false positive report of a malignant sigmoid stricture; four subcentimetre polyps were overlooked. Virtual colonoscopy enabled visualisation of the entire colon in 35 patients; conventional colonoscopy was incomplete in 14 patients. Virtual colonoscopy correctly localised all 38 cancers, compared with 32 using conventional colonoscopy.

Conclusion-Virtual colonoscopy is a feasible method for evaluating the colon; it may have role in diagnosis of colorectal cancer and polyps.

(Gut 1998;43:806-811)
\end{abstract}

Keywords: colonic neoplasms; computed tomography; computer simulation

Over the past three years, simulated endoscopic visualisation using virtual reality imaging has emerged as a promising novel approach to hollow organ evaluation. It is a technique that has been successfully applied to imaging of the airways, sinuses, bladder, and colon. ${ }^{1-7}$ Virtual colonoscopy involves interactive real time manipulation of helical computed tomography (CT) data to simulate conventional colonoscopy. ${ }^{5-9}$ It offers several potential advantages over other techniques for colorectal evaluation: virtual colonoscopy permits a complete evaluation of the whole colon, can be rapidly performed in a single breathhold helical CT acquisition, and does not require either the use of sedation or contrast material administration.

Preliminary in vitro studies have indicated the ability of virtual colonoscopy to show mucosal lesions as small as $4-5 \mathrm{~mm}$ in size, ${ }^{8}$ but few studies to date have addressed the clinical application of virtual colonoscopy in patient material. The aim of this study was to evaluate the ability of virtual colonoscopy to detect colorectal cancers and polyps in patients with endoscopically proven colorectal neoplasms and to correlate the findings of virtual colonoscopy with the results of conventional colonoscopy, surgery, and histopathology.

\section{Materials and methods}

STUDY GROUP

Thirty eight patients ( 23 men, 15 women) with findings on sigmoidoscopy or colonoscopy (Olympus videoendoscopes, Olympus Corporation, Lake Success, New York) suspicious for colorectal carcinoma were prospectively studied. The mean age of the patient group was 57 years (range $41-85$ years). The study protocol was approved by the Institutional Review Board and written informed consent was obtained in all cases.

Twenty nine patients had virtual colonoscopy performed within three hours following a conventional colonoscopy examination that was reported as showing a colorectal carcinoma. Nine patients with a recent diagnosis of colorectal carcinoma made on sigmoidoscopy had virtual colonoscopy performed immediately prior to follow up total colonoscopy. In this latter group of patients, the ability of virtual colonoscopy to detect coexisting colorectal polyps could be evaluated prior to their endoscopic removal.

Indications for endoscopy in our study group included rectal bleeding $(n=13)$, positive faecal occult blood testing $(n=11)$, altered bowel habit $(n=7)$, anaemia of unknown origin $(n=4)$, abdominal pain $(n=2)$, and as part of the work up in a patient with known hepatic metastases.

\section{TECHNIQUE}

All patients had virtual colonoscopy performed on the same day as conventional colonoscopy. Each patient, therefore, received
Accepted for publication 4 February 1998 
a standard colonoscopy bowel preparation, consisting of either 4 litres of polyethylene glycol electrolyte solution (GoLytely; Braintree Laboratories, Braintree, Massachusetts) taken the evening before the examinations or a 48 hour liquid diet combined with $8 \mathrm{oz}$ doses of magnesium citrate and a commercially available bisacodyl and phospha soda preparation kit (Fleet Prep 3; Fleet Pharmaceuticals, Lynchburg, Virginia). Virtual colonoscopy was performed on the same day as conventional colonoscopy in all cases in order to avoid a second bowel preparation.

Virtual colonoscopy examinations were performed according to a standard protocol. Patients were placed in the right lateral decubitus position on the CT table and a rectal enema tube was inserted. Patients were then turned supine and room air gently insufflated into the colon to patient tolerance. One mg of glucagon intravenously (Glucagon, Lilly and Co., Indianapolis, Indiana) was given immediately prior to a helical CT of abdomen and pelvis to allow optimal colonic distension, minimise peristalsis, and alleviate spasm. A standard CT scout film of abdomen and pelvis was acquired to assess the degree of colonic distension, and further air insufflation performed if required. Using the CT scout film, each examination was tailored to encompass the entire colon from caecum to rectum.

All CT examinations were performed using a helical CT scanner (Picker PQ 5000; Picker International, Cleveland, Ohio). Images were acquired using a $5 \mathrm{~mm}$ collimation with a table speed of $6.25 \mathrm{~mm} / \mathrm{s}$ (pitch 1.25), $110 \mathrm{~mA}, 110$ $\mathrm{kVp}$, and a $512 \times 512$ matrix. A single breathhold acquisition was used when possible to encompass the entire colon. Images were reconstructed at $2 \mathrm{~mm}$ intervals, with a $3 \mathrm{~mm}$ slice overlap (reconstruction index of 2). Following the supine scan, the helical CT was repeated with the patient prone.

The CT data were downloaded to an independent workstation (Picker Voxel Q workstation, Picker International, Cleveland, Ohio) equipped with prerelease Picker software (epi-Scope 3.4, Voyager 1). Data segmentation was performed to remove unwanted soft tissue and osseous structures. Using perspective volume rendering algorithms, a retrograde intraluminal "fly-through" navigation through the volume of CT data from rectum to caecum was generated by a single radiologist (HMF) blinded to the results of conventional endoscopy (fig 1). The navigation was repeated in an antegrade direction from caecum to rectum. Both antegrade and retrograde "fly through" virtual colonoscopy studies were stored in a cine loop format and viewed directly from the workstation monitor.

Conventional colonoscopy was performed by a staff gastroenterologist (DPN) on the same morning as virtual colonoscopy. Standard endoscopy equipment was used in each case (Olympus videoendoscopes, Olympus Corporation, Lake Success, New York). The endoscopist was asked to document the presence, location, and size of all polyps and cancers seen on conventional colonoscopy.

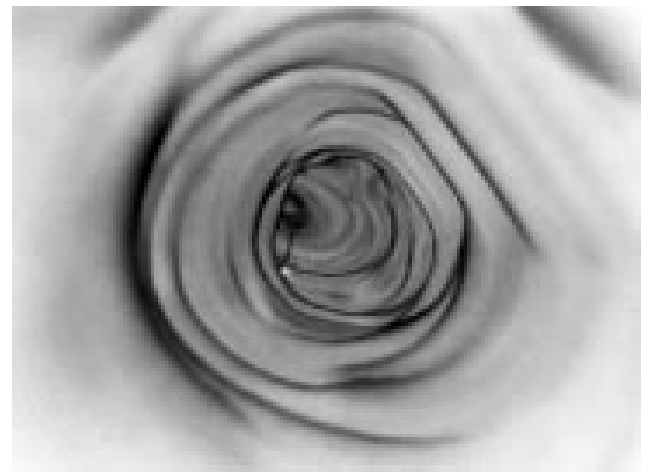

Figure 1 Normal colon. Virtual colonoscopy (retrograde view) showing normal haustral folds and lumen perspective.

\section{INTERPRETATION}

All 38 virtual colonoscopy examinations were reviewed by two experienced gastrointestinal radiologists (JTF, PDC) blinded to the results of the conventional colonoscopy. The virtual colonoscopy studies were reviewed on a 17 inch Picker Voxel Q monitor at a variable frame rate of 5-30 frames per second. Virtual colonoscopy studies generated from the supine CT data acquisitions were used for initial interpretation. Both antegrade (caecum to rectum) and retrograde (rectum to caecum) navigations were evaluated to enable visualisation of both sides of haustral folds. Prone studies were reconstructed when virtual colonoscopy studies obtained from supine CT data were limited by poor distension, retained intraluminal fluid or stool, or when difficulties arose in differentiating polyps or cancers from retained stool.

Note was made of any polyp or mass identified on virtual colonoscopy. Polyps were defined as projections into the colonic lumen measuring less than $2 \mathrm{~cm}$ in maximum dimension, while masses were defined as lesions $2 \mathrm{~cm}$ or greater in size. Both polyps and masses were scored in terms of number, location, size, shape (polypoid, pedunculated, sessile, or annular), and surface appearance (smooth/irregular/ ulcerated). The level of confidence with which the observers reported the presence of a polyp or mass was documented on a scale of $1-5$ ( $1=$ uncertain, $2=$ unlikely, $3=$ possible, $4=$ probable, $5=$ certain).

The results of virtual colonoscopy were correlated with the results of conventional colonoscopy and with the operative findings and histopathological outcome in each case.

\section{Results}

Thirty eight colorectal carcinomas were surgically resected in $37 / 38$ patients. One patient had synchronous tumours involving the sigmoid and transverse colon. One patient, although reported as having a sigmoid stricture suspicious for carcinoma on initial colonoscopy, had a normal follow up double contrast barium enema and a normal repeat colonoscopy. The mean size of the 38 resected colorectal carcinomas was $3.4 \mathrm{~cm}$ (range $2.3-6.5 \mathrm{~cm}$ ). Twelve of the 38 cancers were located in the sigmoid colon, seven in the 
Table 1 Results of conventional and virtual colonoscopy in the detection of pathologically confirmed colorectal masses and polyps

\begin{tabular}{lll}
\hline Technique & Confirmed cancers & Confirmed polyps \\
\hline Conventional colonoscopy & $39(1$ false positive $)$ & $\begin{array}{l}19(4 \text { false negative })^{\star} \\
22(3 \text { false negative, } 2 \text { false } \\
\text { Virtual colonoscopy }\end{array}$ \\
& 38 & positive $)$
\end{tabular}

* Two of the four false negative conventional colonoscopy results were caused by the presence of a distal occlusive carcinoma resulting in failure of the colonoscope to reach the polyp site.

descending colon, four at the splenic flexure, five in the transverse colon, two at the hepatic flexure, three in the ascending colon, and five in the caecum.

All 38 cancers were identified and biopsied at conventional colonoscopy (see table 1). Endoscopists failed to pass proximal to a tumour in nine patients with constricting annular carcinomas. In a further five patients, endoscopists identified a distal colorectal carcinoma but failed to reach the caecum for a variety of reasons unrelated to the presence of pathology. One patient, as described above, was initially diagnosed as having a malignant sigmoid stricture on conventional colonoscopy, but this was subsequently shown to be a false positive result. Conventional colonoscopy correctly predicted the location of 32/38 tumours, with six cancers given a location that was either too proximal or distal compared with the findings at the time of surgery.

On virtual colonoscopy, all 38 carcinomas were identified; each was reported with a confidence level of 5, and all were correctly
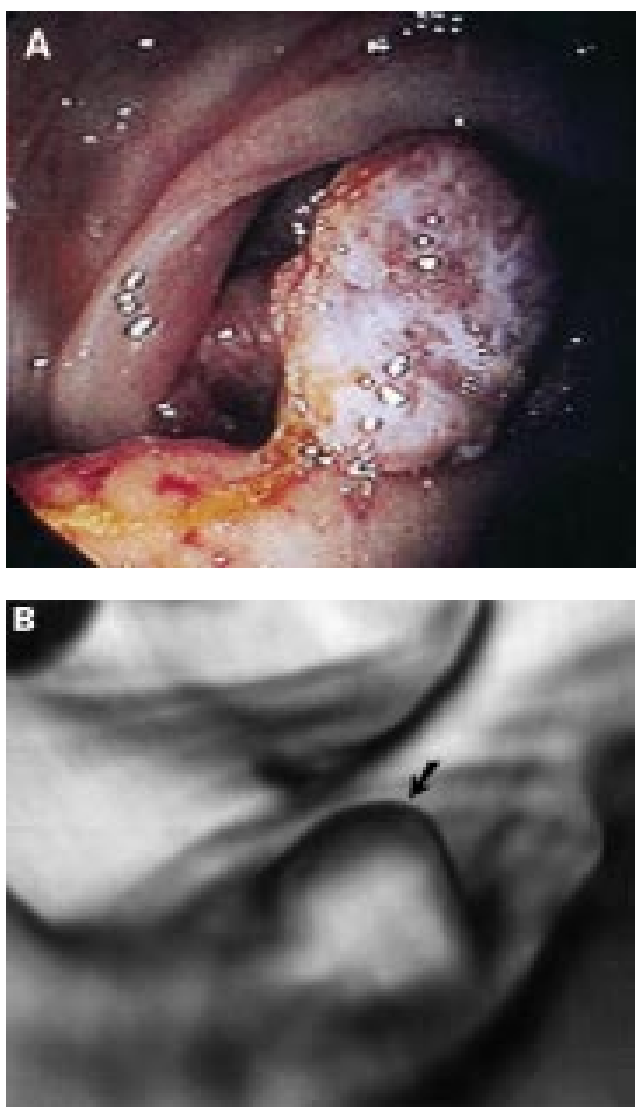

Figure 2 Caecal carcinoma. A $2.5 \mathrm{~cm}$ caecal carcinoma i shown on both conventional $(A)$ and virtual colonoscopy (B) (arrow). Note smoothing of tumour surface detail, a consistent finding on virtual colonoscopy images. localised as judged by findings at surgery (figs 2 and 3). Virtual colonoscopy allowed visualisation of the entire colon to the caecum in 35/38 patients, including the colons of eight of the nine patients with annular constricting carcinomas that could not be passed endoscopically. In three patients, retained stool in one or more segments of colon prevented a complete evaluation to the caecum. In five patients, there was suboptimal distension of the rectosigmoid and ascending colon using the supine data alone but satisfactory virtual colonoscopy examinations were obtained using data from the prone acquisitions.

Twenty three polyps were confirmed histopathologically in the 38 patients. Nineteen of the 23 polyps were identified on conventional colonoscopy, 18 of which were removed endoscopically with one polyp, located adjacent to a carcinoma, remaining in situ and removed with the tumour at the time of surgery. Two of four polyps missed on conventional colonoscopy were located proximal to occlusive carcinomas in segments of colon not visualised endoscopically. Both polyps were identified on the surgical specimen. Two caecal polyps missed on conventional colonoscopy were located on the "blind side" of a haustral fold. Both polyps were subsequently identified on follow up endoscopy with retroflexion of the colono-

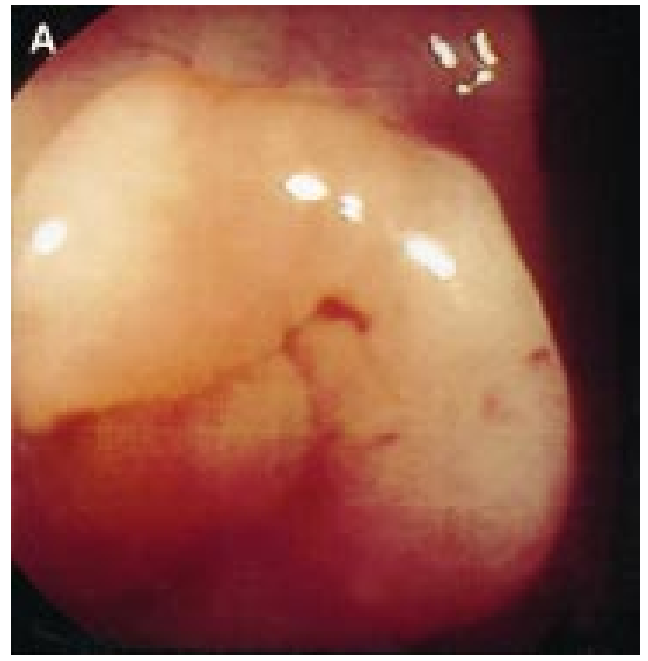

B

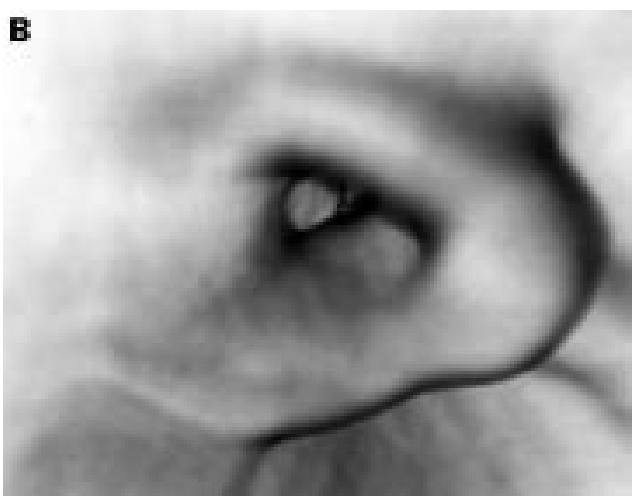

Figure 3 Splenic flexure carcinoma. An annular constricting splenic flexure carcinoma is shown on conventional $(A)$ and virtual colonoscopy (B). Although the tumour could not be passed endoscopically, at virtual colonoscopy the colon proximal to the tumour was clearly visualised and was normal. 

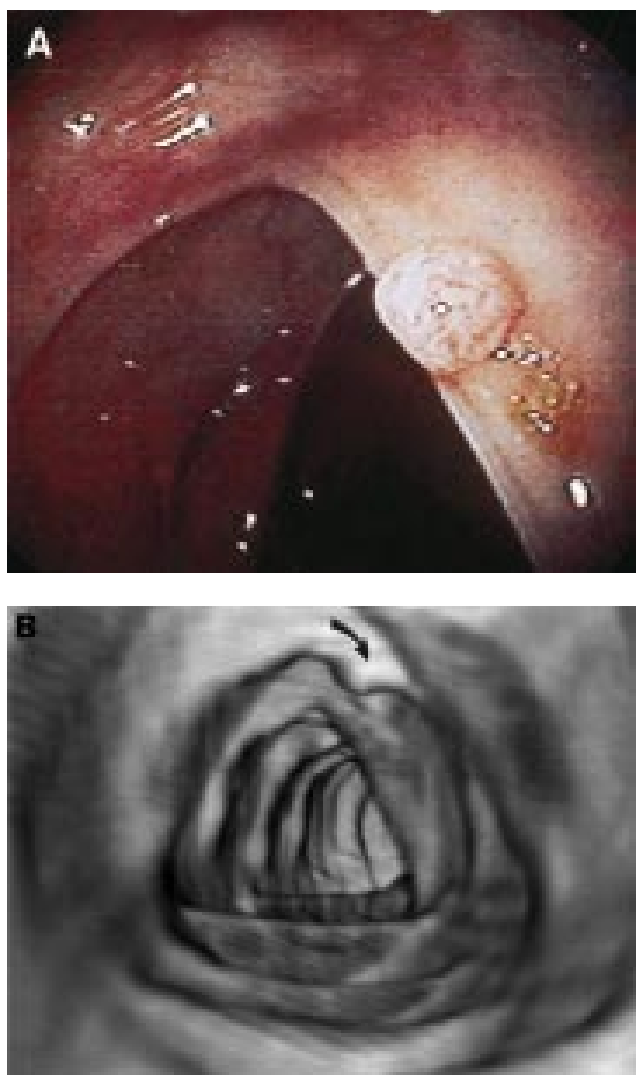

Figure 4 Transverse colon polyp. A 4 mm polyp is seen on both conventional $(A)$ and virtual colonoscopy $(B)$ (arrow). The typical triangular configuration of the transverse colon is readily appreciated on both conventional and virtual colonoscopy images.

scope. The mean size of the 19 polyps identified on conventional colonoscopy (measured against the size of a standard $3 \mathrm{~mm}$ biopsy forceps) was $7 \mathrm{~mm}$ (range 4-12 $\mathrm{mm}$ ). The size of the four "missed" polyps was $6 \mathrm{~mm}$, $7 \mathrm{~mm}, 7 \mathrm{~mm}$, and $10 \mathrm{~mm}$ respectively. Of the total number of polyps $(n=23)$, two were located in the rectum, seven in the sigmoid colon, six in the descending colon, four in the transverse colon, one in the ascending colon, and three in the caecum.

Twenty of 23 polyps were identified on virtual colonoscopy (fig 4). These polyps were found predominantly in the subgroup of patients $(n=9)$ who underwent virtual colonoscopy prior to conventional colonoscopy following an abnormal sigmoidoscopy examination (polyps identified on conventional colonoscopy performed prior to virtual colonoscopy were removed endoscopically and therefore were not available for virtual colonoscopy evaluation). The two polyps located proximal to occlusive carcinomas in segments of colon not visualised on conventional colonoscopy, were identified on virtual colonoscopy and confirmed histopathologically. In addition, the two caecal polyps located on the "blind side" of a haustral fold and missed on the initial conventional colonoscopy examination were identified on virtual colonoscopy. Three polyps measuring 4 $\mathrm{mm}, 6 \mathrm{~mm}$, and $6 \mathrm{~mm}$ respectively, were missed on virtual colonoscopy and there were two false positive findings of polyps (reported as $6 \mathrm{~mm}$ and $8 \mathrm{~mm}$ in size) presumably due to retained stool. Where a false positive diagnosis of pathology was made on virtual colonoscopy, observers reported the abnormality with a lower level of confidence ( 2 or 3 out of a possible 5) compared with true positive results (4 or 5 out of a possible 5).

On average, the total CT room time for virtual colonoscopy was 20 minutes, physician input time for image manipulation was approximately 35 minutes, and the time required for virtual colonoscopy interpretation was 10 minutes.

\section{Discussion}

Simulated endoscopic visualisation (virtual endoscopy) represents the newest and most exciting imaging technique for hollow organ evaluation. Virtual endoscopy was first applied to the colon by Vining et al in 1994 who described the first in vivo virtual colonoscopy examination. ${ }^{9}$ Virtual colonoscopy is a technique made possible only because of recent advances in helical CT technology and high performance computer software programs for image manipulation. By applying specific computer algorithms (perspective volume rendering algorithms) to helical CT data sets, an endoluminal "flight-path" through any hollow organ can be traced, giving the observer a sense of motion through space in a manner similar to conventional endoscopy.

Since virtual colonoscopy was first described a number of similar CT based colon imaging techniques have been developed. ${ }^{10-16}$ Spiral CT pneumocolon, a term used by Amin et al, ${ }^{13}$ describes dynamic intravenous contrast enhanced thin section helical CT of the air insufflated colon with aid of smooth muscle relaxants. CT data are displayed and interpreted in the conventional manner without additional postprocessing and without the interactive capabilities used for virtual colonoscopy. Amin et al successfully applied this technique in four patients with colorectal cancer, but have not since reported its value in colorectal polyp detection. Hara et al of the Mayo Clinic successfully devised the method of CT colography, ${ }^{14-16}$ a term that describes thin section CT of the colon ( $5 \mathrm{~mm}$ collimation, $5 \mathrm{~mm}$ table incrementation, and $1 \mathrm{~mm}$ reconstruction intervals) displayed as multiple reformatted two dimensional CT images at cross sectional and orthogonal angles to the long axis of the colon, supplemented by extraluminal three dimensional depth shaded volume renderings and endoluminal three dimensional renderings of the colon. Using CT colography, these researchers evaluated 30 endoscopically proven polyps in 10 patients and detected $100 \%$ of all polyps greater than 1 $\mathrm{cm}$ in diameter, $71 \%$ of polyps between 0.5 and $0.9 \mathrm{~cm}$, and up to $28 \%$ of polyps greater than $0.5 \mathrm{~cm}$ in size. This was the first in vivo study of CT for colon polyp detection, and their results represent the best reported to date.

The term which we use is virtual colonoscopy which specifically refers to interactive real time navigations through the colon directly simulating conventional colonoscopy ${ }^{5-7}$ Unlike 
spiral CT pneumocolon and CT colography, virtual colonoscopy examinations are viewed as a single cine loop display without the need for additional multiplanar two dimensional and static three dimensional reconstructions. Using this technique, Dachman et al evaluated the sensitivity of virtual colonoscopy in the detection of simulated polyps in pig colon and, using optimum scan parameters, successfully visualised 3, 7, and $10 \mathrm{~mm}$ polyps respectively. ${ }^{8}$ Rubin et al successfully used perspective volume rendering for virtual colonoscopy in one patient with a constricting ascending colon carcinoma and, using the same technique, successfully showed a $5 \mathrm{~mm}$ adenomatous polyp. ${ }^{10}$ Although numerous authors have reported their preliminary results of in vivo studies of virtual colonoscopy at scientific meetings, little published data exist regarding the clinical efficacy of virtual colonoscopy at this time.

Regardless of the technology and terminology used, the clinical protocols for performance of each of these CT techniques are essentially the same. ${ }^{6}$ All CT colon techniques require thorough bowel cleansing, air insufflation of the colon, smooth muscle relaxation, helical CT acquisition, and some form of computer postprocessing. The primary difference between the techniques described to date is the manner in which the helical CT data are manipulated and displayed. Using the technique which we describe for virtual colonoscopy, all 38 cancers and all polyps measuring greater than $6 \mathrm{~mm}$ in size were identified. In addition, virtual colonoscopy enabled visualisation of the entire colon in 35 of the 38 patients and correctly localised all 38 cancers, compared with 32 cancers that were correctly localised using conventional colonoscopy alone.

Specific aspects of this study merit further brief discussion. Although four of 23 polyps $(16 \%)$ were missed on conventional colonoscopy, two of the four polyps were located proximal to constricting carcinomas in segments of bowel not accessible to endoscopy. This "miss rate" compares favourably with recent published data that indicate a failure rate of $24 \%$ for colorectal polyp detection at conventional colonoscopy. ${ }^{17}$ Furthermore, as many of the polyps identified on conventional colonoscopy had been removed prior to virtual colonoscopy, polyp detection rates in our study strongly favour the CT technique. Most lesions left behind after colonoscopy were either sizeable cancers or missed polyps. As this study had no control patients and radiologists were biased by the knowledge that a significant lesion was already present, the true sensitivity and specificity of virtual colonoscopy cannot be estimated from our results. The results of this study cannot be extrapolated to a screening population where the pretest probability of disease is very different. Despite these biases, the ability to identify and locate colorectal cancers and polyps correctly with a high degree of confidence using virtual colonoscopy is impressive.

There are numerous potential benefits of virtual colonoscopy over conventional colonoscopy. Virtual colonoscopy is, for the patient, a "60 second" examination, without requirement for anaesthesia or sedation. It is non-invasive, without the small but definite risk (approximately $0.075 \%{ }^{18}$ ) of perforation associated with conventional colonoscopy. It represents, therefore, a more attractive alternative to conventional colonoscopy in elderly or infirm patients. In our experience, it provides a more complete examination of the colon in a greater number of patients than is possible with conventional colonoscopy, consistently shows the colon proximal to annular constricting carcinomas, and more accurately predicts tumour location than is possible with conventional colonoscopy. Virtual colonoscopy does not have the limitation of view direction. By providing both direct antegrade and retrograde visualisation, virtual colonoscopy allows detection of polyps located on the "blind side" of haustral folds, which can only be seen endoscopically with retroflexion of the endoscope.

Limitations of virtual colonoscopy in the evaluation of colorectal disease are many. ${ }^{56}$ Retained stool may both mimic and disguise colorectal pathology. Areas of poorly distended colon cannot be evaluated and may even mimic colorectal strictures. On the other hand, overinflation can lead to troublesome small bowel reflux of air leading to difficulties in navigating the colon and artefactual lucencies in the bowel wall. Flat lesions are difficult to detect and subtle alterations in mucosal colour and texture are not shown. Fine surface detail is substantially smoothed, such that distinction between mucosal and submucosal, and benign and malignant colonic masses is difficult. Although the patient radiation dose per acquisition is low (0.44 rem, approximately equal to two plain abdominal radiographs), ${ }^{6} 16$ virtual colonoscopy should be avoided in pregnant women and children for obvious reasons. Finally, and most importantly, virtual colonoscopy, at best, serves only to detect the presence of disease. Conventional colonoscopy is required in all patients with abnormal virtual colonoscopy studies to confirm findings and for biopsy.

There are numerous, more global, limitations to the widespread use of virtual colonoscopy for colorectal cancer and polyp detection. ${ }^{6} 7$ Although our results and those of others are promising, estimation of the true accuracy of virtual colonoscopy will require large scale, prospective, multicentre trials comparing virtual with conventional colonoscopy in patients at risk for but not having an established diagnosis of colorectal cancer. Such an undertaking is currently hampered by a lack of standardisation in terms of terminology, performance, and interpretation of virtual colonoscopy studies. Because virtual colonoscopy is a technique in evolution, many different computer systems are currently being evaluated and, as yet, there is no consensus agreement as to which is best. Careful clinical outcome and cost effectiveness studies will need to be performed to assess the status of virtual colonoscopy compared with existing practices of barium enema and conventional colonoscopy. At this time, virtual colonoscopy remains expensive both in terms of cost and time. Although the cost of dedicated software 
and hardware required for virtual colonoscopy has decreased considerably, data manipulation currently demands significant physician input time and training. Balanced against this is the need to provide virtual colonoscopy at a cost that is competitive with conventional diagnostic colonoscopy. In a climate of increasing financial restraint and reductions in reimbursement, virtual colonoscopy may or may not prove to be a realistic alternative to diagnostic colonoscopy or barium enema.

The technology of virtual endoscopy is rapidly evolving. Software programs are now available that permit automated navigations, substantially reducing the time required for image reconstruction. "Intelligent" computer software has been developed that automatically alerts the radiologist to the presence of pathology by identifying a change in colonic wall thickness. The feasibility of magnetic resonance colonoscopy has recently been reported, ${ }^{19} 20$ another evolving technique against which CT virtual colonoscopy must be compared. Whether or not virtual colonoscopy ever becomes a "reality" will depend not only on the accuracy of the technique, but on the speed, availability, and cost of a variety of computer techniques currently under investigation.

The American Cancer Society (ACS) recently revised its guidelines for colorectal cancer screening. ${ }^{21}$ The most significant change in its screening recommendations involved the novel concept of the total colon examination, referring either to total colonoscopy or double contrast barium enema for "average" and "moderate" risk patients, starting at age 50 years. In its revised recommendations, the American Cancer Society recognised the potential for future use of emerging CT techniques including virtual colonoscopy for colorectal cancer screening. Should virtual colonoscopy or any of the related CT techniques be confirmed as reliable, accurate, and cost effective methods for examining the total colon, it is possible that they will have a role in screening for colorectal cancer and may impact on future screening recommendations.

\section{Conclusion}

This study shows that virtual colonoscopy is a feasible method for detecting colorectal neoplasms. We used commercially available equipment now widely installed in the USA and in many European countries and detected all colorectal neoplasms greater than $6 \mathrm{~mm}$ in size with a high degree of confidence. Furthermore, this technique correctly localised all cancers and polyps and provided a more complete evaluation of the colon than was possible with conventional colonoscopy. The challenge remains to define better the diagnostic accuracy of this technique in a screening population.

1 Vining DJ. Virtual endoscopy: is it reality? Radiology 1996;200:30-1.

2 Kauczor H-U, Wolcke B, Fischer B, et al. Threedimensional helical CT of the tracheobronchial tree: evaluation of imaging protocols and assessment of suspected stenoses with bronchoscopic correlation. AfR Am $\mathcal{F}$ Roentgenol 1996;167:419-24.

3 Summers RM. Navigational aids for real-time virtual bronchoscopy. AfR Am f Roentgenol 1997;168:1165-70.

4 Fenlon HM, Bell T, Hussain S. Virtual cystoscopy: early clinical experience. Radiology 1997;205:272-5.

5 Royster AP, Fenlon HM, Clarke PD, et al. Threedimensional "virtual reality" techniques with colonoscopic dimensional "virtual reality" techniques with colonoscop

6 Fenlon HM, Ferrucci JT. Virtual colonoscopy-what will Fenlon HM, Ferrucci JT. Virtual colonoscopy-what
the issues be? AfR Am $\mathcal{f}$ Roentgenol 1997;169:453-8.

7 Kay CL, Evangelou HA. A review of the technical and clinical aspects of virtual endoscopy. Endoscopy 1996;28:76875 .

8 Dachman AH, Lieberman J, Osnis RB, et al. Small simulated polyps in pig colon: sensitivity of CT virtual colography. Radiology 1997;203:427-30.

9 Vining DJ, Gelfand DW, Bechtold RE, et al. Technical feasibility of colon imaging with helical CT and virtual reality [abstract]. AfR Am f Roentgenol 1994;162(suppl):104.

10 Rubin GD, Beaulieu CF, Argiro V, et al. Perspective volume rendering of CT and MR images: applications for rendering of CT and MR images: applicatior
endoscopic imaging. Radiology 1996;199:321-30.

11 Rubin GD, Napel S, Leung AN. Volumetric analysis of volumetric data: achieving a paradigm shift. Radiology 1996;200:312-17

12 Johnson PT, Heath DG, Bliss DF, et al. Three-dimensional $\mathrm{CT}$ : real-time interactive volume rendering. $A \mathcal{F R} A m^{f}$ Roentgenol 1996;167:581-3.

13 Amin Z, Boulos PB, Lees WR. Technical report: spiral CT pneumocolon for suspected colonic neoplasms. Clin Radiol 1996;51:56-61.

14 Hara AK, Johnson CD, Reed JE, et al. Colorectal polyp detection with CT colography: two- versus threedimensional techniques (work in progress). Radiology 1996;200:49-54.

15 Hara AK, Johnson CD, Reed JE, et al. Detection of colorectal polyps by computed tomographic colography: feasibility of a novel technique. Gastroenterology 1996;110:284-90.

16 Hara AK, Johnson CD, Reed JE, et al. Reducing data size and radiation dose for CT colonography. AfR Am $\mathcal{F}$ Roentgenol 1997;168:1181-4

17 Rex DK, Cutler CS, Lemmel GT, et al. Colonoscopic miss rates of adenomas determined by back-to-back colonoscopies. Gastroenterology 1997;112:24-8.

18 Farley DR, Bannon MP, Zietlow SP, et al. Management of colonoscopic perforations. Mayo Clin Proc 1997;72:72933.

19 Schoenenberger AW, Bauerfield P, Krestin GP, et al. Virtual colonoscopy with magnetic resonance imaging: in vitro colonoscopy with magnetic resonance imaging: in vitro
evaluation of a new concept. Gastroenterology 1997;112: 1863-70.

20 Ahlquist DA, Johnson CD. Screening in cyberspace: the next generation? Gastroenterology 1997;122:2150-2.

21 American Cancer Society guidelines for screening and surveillance for early detection of colorectal polyps and cancer. Update 1997. CA, Cancer F Clin 1997;47:154-60. 